\title{
Automation of Fingerprint Recognition Using OCT Fingerprint Images
}

\author{
Nasibe Akbari, Ali Sadr \\ Iran University of Science and Technology, Tehran, Iran. \\ Email: nakbari@elec.iust.ac.ir, sadr@iust.ac.ir \\ Received February $14^{\text {th }}, 2011$; revised August $12^{\text {th }}, 2011$; accepted November $9^{\text {th }}, 2011$
}

\begin{abstract}
Automated recognition of a person is one of the most critical issues in the modern society. Common biometric systems rely on the surface topography of an object and, thus, are potentially vulnerable for spoofing. Optical coherence tomography is a technology that has the capability to probe the internal structure of multilayered tissues. The paper describes an algorithm for automation fingerprint recognition that the algorithm is applied on the OCT fingerprint images. This algorithm is based on scanning of the enhanced and segmented OCT images.
\end{abstract}

Keywords: Optical; Coherence; Tomography; Speckle Denoising; Fingerprint Recognition

\section{Introduction}

Automated or semi-automated recognition of a person is one of the critical issues. Fingerprint recognition is one of the most commonly used methods of biometrics. Every person is known to have unique, immutable series of ridges and furrows on the surface of the finger, called fingerprint [1]. Fingerprint recognition is cheap and relatively robust biometric method and it has been extensively studied and applied for many popular applications. These methods rely on the surface topography of the finger and thus are vulnerable for spoofing with using artificial fingerprint [2-4]. During the past several years, significant improvements have been made by several scientific groups to enhance the robustness of the fingerprint readers based on the recognition of the surface topology. Optical Coherence Tomography (OCT) technique for noninvasive identification of artificial materials used for spoofing. A typical time-domain OCT system is based on the Michelson interferometer configuration with a low coherent laser in a source arm, a moving mirror in a reference arm, an object under study in a sample arm, and a photodetector to measure the interference signal in a detection arm. Due to use of partially coherent light in OCT, speckle noise is an inherent component of any OCT tomogram. Presence of speckle noise results in granular appearance of the image, which in turn can obscure small or low reflectivity features, thus degrading the image quality of OCT tomograms. Furthermore, it can impede or limit the performance of image segmentation and pattern recognition algorithms that are used to extract, analyze, and recognize diagnostically relevant features. In this paper we explain two methods for automation identification of finger gummy with embedded artificial layer. Thus for recognition of artificial layer must be reduce speckle noise. At the first the OCT fingerprint images is enhanced. Image enhancement is containing: noise removal, edge detection and segmentation. The prepared segmented image is scanned for identification of additional layer.

\section{Materials and Methods}

\subsection{Experimental Setup}

Figure 1 shows a schematic of a time-domain OCT system used in these studies (Imalux Corp., Cleveland, Ohio). A low-coherent superluminescent laser diode with a wavelength of $1300 \pm 15 \mathrm{~nm}$ and an output power of $375 \mu \mathrm{W}$ was used as the optical source in this system. Light in the sample arm of the interferometer was directed into tissues using a single-mode optical fiber and a specially designed miniature endoscopic probe. The endoscopic probe allowed for the latera scanning of the sample surface in the lateral direction (axis). Light scattered from the sample and light reflecte from the reference arm mirror formed an interferogram which was detected by a photodiode.

In-depth scanning was produced electronically by piezoelectric modulation of the fiber length. Two-dimensional images were obtained by scanning over the sample surface in the lateral direction ( $\mathrm{X}$ axis) and in-depth ( $\mathrm{Z}$ axis) scanning by the interferometer. The acquired images were $450 \times 450$ pixels. 


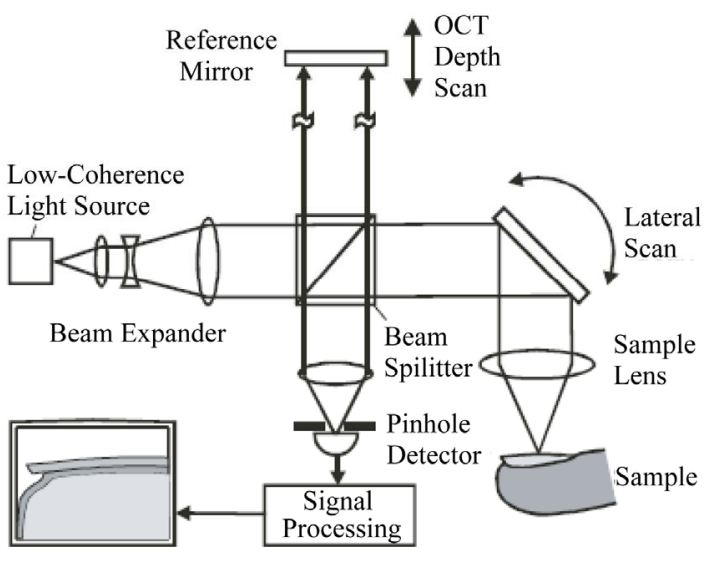

Figure 1. Schematic of the OCT system used in these studies. PD, photo detector; ADC, analog-to-digital converter [5].

\subsection{Materials}

Several materials that could be used for spoofing fingerprint biometric system have been studied. For instance gelatin, silicon, waxes of different concentration. Gelatin sample were prepared by dissolving solid gelatin in water. The solutions were heated and then left out for solidification. There are slices with different thickness. A finger was carefully washed with soap to get rid of the dust and tissue oil. Then, the finger was pressed firmly into the plasticin to leave the fingerprint pattern. Hence the artificial fingerprint dummy was ready for the experiments.

\subsection{Experimental Protocol}

In the first part of experiment, OCT images of a thumb were taken to obscure characteristics layer of the skin: stratum corneum of epidermis, epidermis, dermis (Figure 2(a)). In the next phase, artificial fingerprint dummy were placed on the finger and OCT image was taken. (Figure 2(b))

\subsection{Automation}

Automated or semi-automated recognition of a person is the most critical issues. It has been shown the biometric imaging systems based on fingerprint Recognition could be easily spoofed with about $\$ 10$ Worth of household supplies. Biometric fingerprint devices have to be more secure, accurate and robust.

Against different fraudulent methods including dummy fingerprints. Optical coherence tomography is capable to identification of artificial materials used to swindle fingerprint biometric devices. There is one method for automated recognition of a fingerprint that it is faked or not. This method is scanning of image for finding edge between artificial layer and real skin.

For the scanning of image, the image must be enhanced and segmented to edge between artificial layer and real

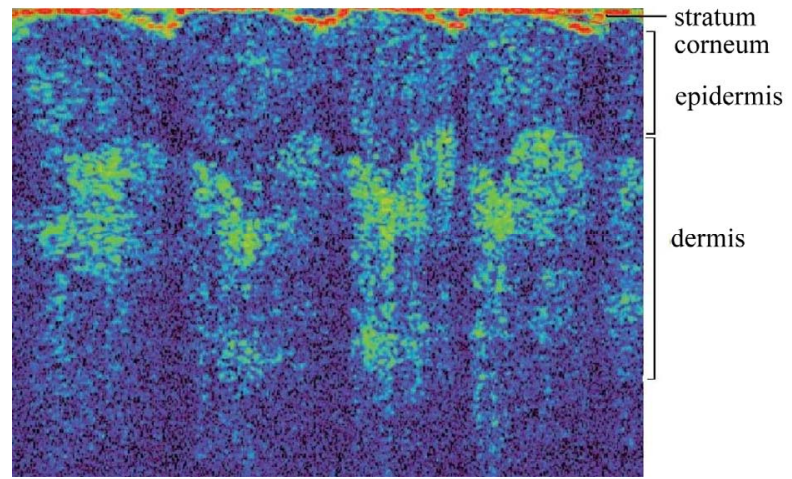

(a)

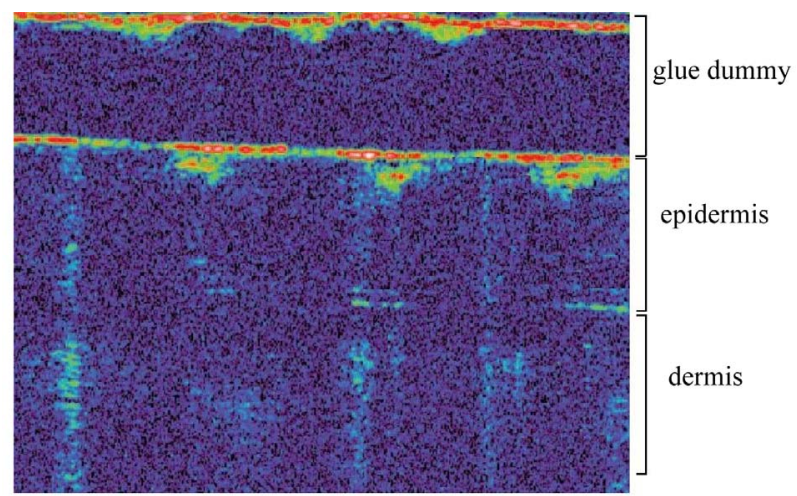

(b)

Figure 2. Two dimensional OCT image from (a) finger skin (b) finger with embedded artificial layer.

skin if it is existence, detected. Then the segmented image is prepared to scanning.

\subsubsection{Noise Removal}

Since OCT is based on low-coherence interferometry, which utilizes the spatial and temporal coherence properties of optical waves backscattered from biological tissue [5]. Due to use of partially coherent light in OCT, speckle noise is an inherent component of any OCT tomogram. Presence of speckle noise results in granular appearance of the image, which in turn can obscure small or low reflectivity features, thus degrading the image quality of OCT tomograms. Furthermore, it can impede or limit the performance of image segmentation and pattern recognition algorithms that are used to extract, analyze, and recognize diagnostically relevant features. Rotating kernel transformation (RKT) [6] were applied to OCT security images to reduce noises.

The RKT technique operates by filtering an image with a set of templates (kernels), and retaining the largest filter output at each pixel. In this technique the input image is convolved with a kernel that is rotated discretely in small steps through 360 . The convolution can be written as [7].

$$
\mathrm{S}(\mathrm{x} ; \mathrm{y})=\mathrm{I}(\mathrm{x} ; \mathrm{y}) * \mathrm{~K}(\mathrm{x} ; \mathrm{y})
$$


where $\mathrm{I}$ is the input image, and $\mathrm{K}_{\theta}$ is the kernel oriented at rotation angle $\theta$ the maximum values are calculated over all rotated kernels, and the output image is defined as

$$
\mathrm{O}(\mathrm{x} ; \mathrm{y})=\operatorname{maximum}\left\{\mathrm{S}_{\theta}(\mathrm{x} ; \mathrm{y}): 0<\theta<360\right\}
$$

RKT were applied to OCT fingerprint images with kernel size: 31 . The larger kernel size causes the speckle in the image is blurred and the edges are less visible but we would like to preserve the edge while at the same time reduce the speckle. In addition, we applied the RKT algorithm to the OCT images sequentially two times and result will be better. Thereby, for the best do noising result, were applied RKT with kernel size 31 and it is repeated.

\subsubsection{Edge Based Segmentation}

After speckle removal for detecting of artificial layer was applied edge detection technique. Vertical gradient is a technique for edge detection. This edge detector was applied to RKT processed image to detect the border between dummy and real skin. in order to decide whether an edge has been found, we applied thresholding for segmentation of image. Thresholding is a common region extraction method. Common thresholding is based on image $\mathrm{s}$ histogram. Since this may be computationally expensive, and image histograms may not have clearly defined valley points, often making the selection of an accurate threshold difficult but also we would like to edge detection is automated while this method based on image histogram then for every image amount of thresholding have been changed. Hysteresis thresholding overcomes this limitation. The algorithm uses 2 thresholds, Thigh, Tlow .

- A pixel $(x, y)$ is called strong if intensity amount of $(\mathrm{x}, \mathrm{y})>\mathrm{T}_{\text {high }}$.

- A pixel $(x, y)$ is called weak if intensity amount of $(\mathrm{x}, \mathrm{y})<\mathrm{T}_{\text {low }}$.

- All other pixels are called candidate pixels.

In each position of $(x, y)$, discard the pixel $(x, y)$ if it is weak, output the pixel if it is strong. If the pixel is a candidate, follow the chain of connected local maxima in both directions along the edge, as long as, amount of ( $\mathrm{x}$, $y)>T_{\text {low }}$. If the starting candidate pixel $(x, y)$ is connected to a strong pixel, output this candidate pixel; otherwise, do not output the candidate pixel.

\subsubsection{Scanning of Image for Fingerprint Recognition}

When artificial layer (if it is existence) is detected by above algorithm, for automation of this systems must to scan the line (edge between artificial layer and real skin). There is one algorithm for to do it. This algorithm constitutes several steps.

Step 1: the thresholded image is containing unwanted signal that may reduces precision of the process, therefore median filtering was applied to remove them.

Step 2: enhanced image is binary. Middle pixels of images are selected to scanning. Since artificial layer is at middle of image and no need to whole of the image is scanned because it is consuming time.

Step 3: at the first of every row, the entire column of image matrix is scanned; if the white pixel is found for the column the next row is tested if it is white the number of row is saved. it is a loop and continues. All of point was accepted with $1 \%$ or $2 \%$ tolerance. This algorithm was repeated until the line is detected.

The number of row with $1 \%$ or $2 \%$ tolerance is the height of artificial layer. If the height is none zero thereby the artificial layer is existence, and fingerprint is faked. But if the height is zero, then the fingerprint is ok.

\section{Result}

\subsection{Noise Removal}

Nonlinear filtering such as rotating kernel transformation (RKT) with kernel size 31 was applied to OCT fingerprint images. This filtering with larger kernel size, the speckle in the image is blurred and the artificial layer edge is more clearly emphasized. Figure 3(a) demonstrates applying RKT with kernel size 31 to Figure 2(a). When the RKT application applied to the OCT images, twice, amplifies the effects of a single application and results in even more smoother images. Figure 3(b) shows the results.

\subsection{Edge Detection and Image Segmentation}

For detecting the boundary of artificial layer and real skin we applied vertical gradient. When, this edge detector were applied to the RKT processed images, detection of edge will be better [8] when vertical gradient were applied to double RKT processed image, detection of line is more visible and the line is continuous. These results are shown in Figure 4. Detection of artificial layer and real skin border by hysteresis thresholding technique is shown in Figure 5. Making the assumption that important edges should be along continuous curves in the image allows us to follow a faint section of a given line and to discard a few noisy pixels that do not constitute a line but have produced large gradients. Therefore we begin by applying a high threshold. These marks out the edges we can be fairly sure are genuine. Starting from these, using the directional information derived earlier, edges can be traced through the image. While tracing an edge, we apply the lower threshold, allowing us to trace faint sections of edges as long as we find a starting point. Figure 5(a) demonstrates result of applying hysteresis thresholding to RKT Processed image. When hysteresis thresholding were applied to twice RKT processed image 


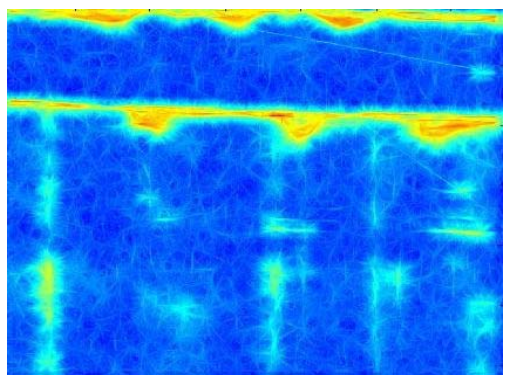

(a)

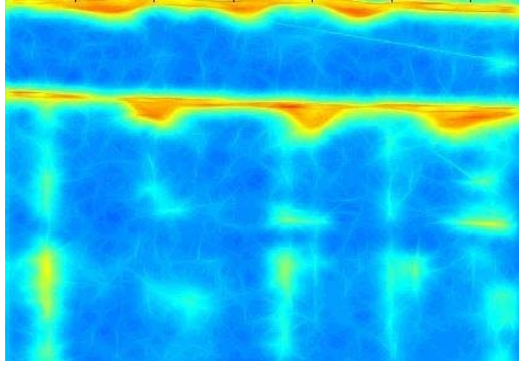

(b)

Figure 3. RKT processing applied to Figure 2(b) with kernel size 31, (a) Once application; (b) Twice application.

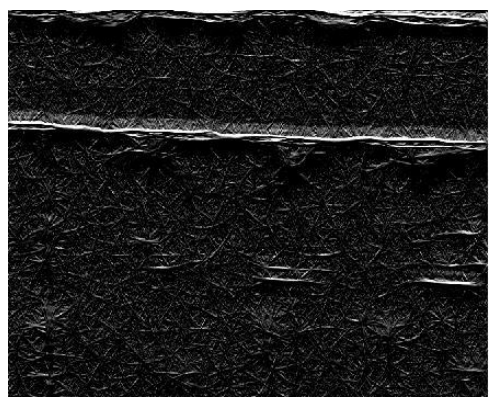

(a)

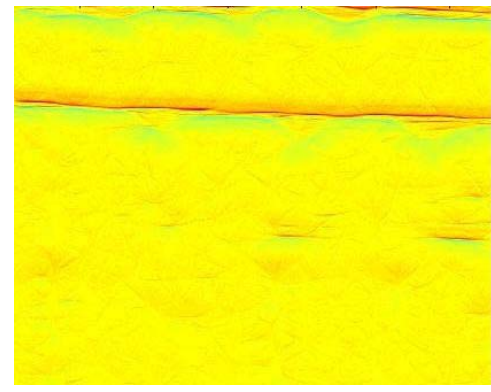

(b)

Figure 4. Vertical gradient applied to (a) RKT processed image; (b) Twice RKT processed image.

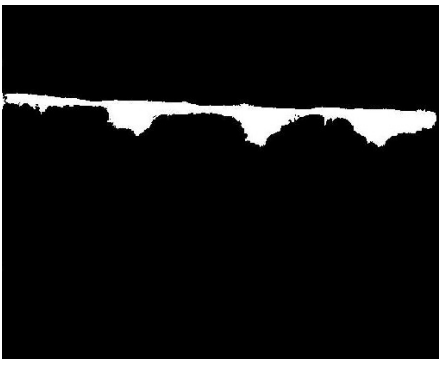

(a)

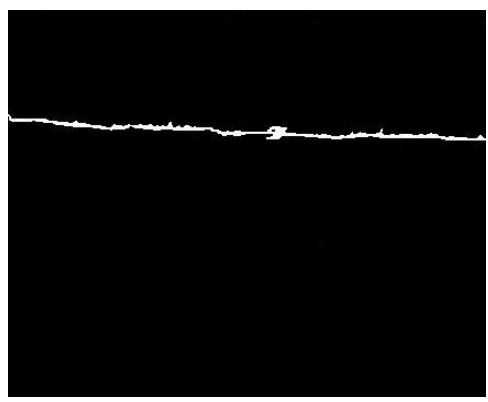

(c)

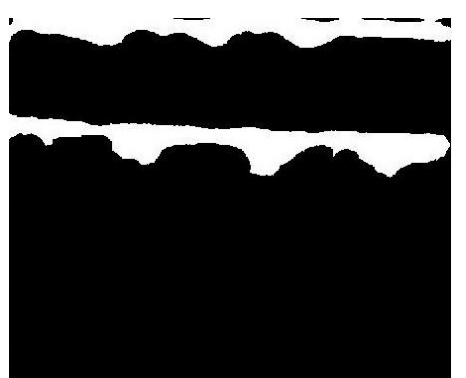

(b)

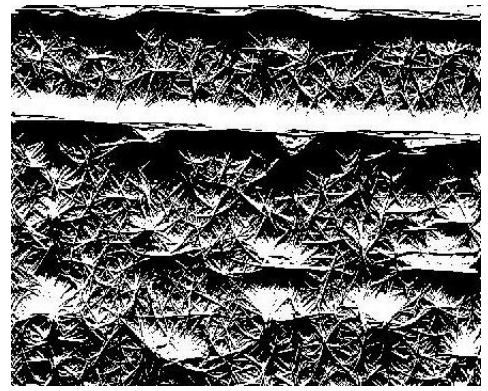

(d)

Figure 5. Result of Hysteresis thresholding for detection of the artificial layer and real skin border performed on (a) RKT 31 processed image; (b) Twice RKT 31 processed image; (c) Vertical gradient of RKT processed image from Figure 4(a); (d) Vertical gradient of twice RKT processed image from Figure 4(b).

(Figure 5(b)), the result is similar to Figure 5(a). However the detected line is so clear and sharp, but width of its is wide and reduces precision of measurement of height of artificial layer. Figure 5(c) demonstrates result of hysteresis thresholding performed on vertical gradient of RKT with kernel size 31 processed image, the detected edge is better since it is sharp and one pixel width, thereby measurement of additional layer is precision.

Figure 5(d) demonstrates result of applying hysteresis thresholding to vertical gradient twice RKT with kernel 
size 31 since double RKT application preserves most of features, therefore when hysteresis thresholding applied to it, despite detection of middle edge, also the features of image will be revealed. That is no optimal for automation.

\subsection{Scanning of Image}

After the enhanced binary image was obtained, for detecting middle line (he edge between artificial layer and real skin), for each row of the intensity matrix, the entire column were scanned. If the continuous line as long as $80 \%$ length of total number of column was detected, the OCT system beeps and an error with this concept: fingerprint is not ok is declared, otherwise operation is continued and it is typing fingerprint is ok.

\section{Discussion}

For automation of fingerprint recognition, we introduce one algorithm. The automation is based on scanning of the image for finding continuous line (the edge between of artificial layer and real skin), thereby the OCT fingerprint image must be enhanced and the unwanted signal should be reduced. For noise removal, rotating kernel transformation with kernel size 31 was performed on to the OCT fingerprint once and more time again (Figure 3). For edge detection we applied vertical gradient to the RKT processed image. When vertical gradient were applied to the double RKT processed image, the artificial layer and real skin boundary is more detectable, while suppressing a noisy background. Also with this method the edge between artificial layer and real skin is continuous and one-pixel width (Figure 4). In order to decide whether an edge has been found, we have applied hysteresis thresholding for segmentation of image (Figure 5) for recognition of the fingerprint that weather it is faked by artificial layer or not, the segmented OCT image is scanned for finding continuous line with white pixel if it is existed.

\section{Conclusions}

OCT systems have been shown to be effective for reli- able fingerprint identification by high resolution imaging of internal structures. In this study, we investigated the method for automation of fingerprint recognition. This method is based on scanning of the segmented OCT image.

At the first, the OCT images were enhanced and segmented, and finally, the images were scanned. Our results showed this approach can achieve significant improvement in automation of fingerprint recognition.

\section{REFERENCES}

[1] S. Prabhakar, S. Pankanti and A. K. Jain, "Biometric Recognition: Security and Privacy Concerns," IEEE Security \& Privacy, Vol. 1, No. 2, 2003, pp. 33-42. doi:10.1109/MSECP.2003.1193209

[2] D. Maltoni, D. Maio, A. K. Jain and S. Prabhakar, "Handbook of Fingerprint Recognition," Springer, Berlin, 2003.

[3] P. R. Vizcaya and L. A. Gerhardt, "Nonlinear Orientation Model for Global Description of Fingerprints," Pattern Recognition, Vol. 29, No. 7, 1996, pp. 1221-1232. doi:10.1016/0031-3203(95)00154-9

[4] T. Matsumoto, H. Matsumoto, K. Yamada and S. Hoshino, "Impact of Artificial Gummy Fingers on Fingerprint Systems," Proceedings of SPIE, Vol. 4677, 2002, pp. 275-289. doi: $10.1117 / 12.462719$

[5] J. M. Schmitt, S. H. Xiang and K. M. Yung, "Speckle in Optical Coherence Tomography," Journal of Biomedical Optics, Vol. 4, No. 1, 1999, pp. 95-105.

[6] J. Rogowska and M. E. Brezinski, "Evaluation of the Adaptive Speckle Suppression Filter for Coronary Optical Coherence Tomography Imaging," IEEE Transactions on Medical Imaging, Vol. 19, No. 12, 2000, pp. 1261-1266.

[7] Y.-K. Lee and W. T. Rhodes, "Nonlinear Image Processing by a Rotating Kernel Transformation," Optics Letters, Vol. 15, No. 23, 1990, pp. 1383-1385. doi:10.1364/OL.15.001383

[8] R. K. Manapuram, M. Ghosn and K. V. Larin, "Identification of Artificial Fingerprints Using Optical Coherence Tomography Technique," Asian Journal of Physics, Vol. 15,2006 , pp. 15-27. 